Rol es of myof i brobl ast s and notch and hedgehog si gnal ing pat hways i $n$ the for mat i on of i nt rahepat i c bi le duct l esi ons i $n$ pol ycyst i c ki dney $r$ at s

\begin{tabular}{|c|c|}
\hline 著者 & 古坊 真一 \\
\hline 著者別表示 & Fur ubo Shi ni chi \\
\hline $\begin{array}{l}\text { jour nal or } \\
\text { publ i cat i on title }\end{array}$ & 博士論文要旨Abst r act および要約Out I i ne \\
\hline 学位授与番号 & 13301乙第2047号 \\
\hline 学位名 & 博士 (医学) \\
\hline 学位授与年月日 & $2013-12-31$ \\
\hline URL & ht t p: //hdl . handl e. net /2297/39436 \\
\hline
\end{tabular}




\section{Roles of myofibroblasts and Notch and Hedgehog signalling pathways in formation of intrahepatic bile duct lesions of the polycystic kidney rat}

Shinichi Furubo, ${ }^{1)}$ Yasunori Sato, ${ }^{1)}$ Kenichi Harada, ${ }^{1)}$ and Yasuni Nakanuma ${ }^{1)}$

${ }^{1)}$ Department of Human Pathology, Kanazawa University Graudate School of Medicine, Kanazawa, Japan

Short title: Notch signalling pathway in PCK rats

Address correspondence to: Yasuni Nakanuma, M.D

Department of Human Pathology

Kanazawa University Graduate School of Medicine

Kanazawa 920-8640, Japan

Tel: +81-76-265-2197 (Japan) Fax: +81-76-234-4229 (Japan)

E-mail: pbcpsc@kenroku.kanazawa-u.ac.jp

Disclosures: None of the authors have any conflict of interest or funding to disclose. 


\begin{abstract}
Objectives: PCK rats, an animal model of Caroli's disease, show a dilatation of intrahepatic bile ducts (IHBD) called "ductal plate malformation". Mesenchymal cells and the Notch and Hedgehog signalling pathways in portal tracts are reportedly involved in the normal development of IHBD, though there have been no studies on the roles of these signalling pathways in PCK rats. Methods: We examined mainly immunohistochemically the expression of the molecules related to these signalling pathways in portal tracts. Results: All molecules related to these signalling pathways expressed in portal tracts in SD rats (control), were also expressed in PCK rats. Mesenchymal cells (myofibroblasts) were frequently found in the connective tissue of portal tracts of SD and PCK rats at E20D, 1D and 1W, and were abundant in PCK rats. Interestingly, myofibroblasts almost disappeared at $3 \mathrm{~W}$ in both strains. Jagged 1 was expressed in mesenchymal cells in portal tracts, and was abundant in PCK rats. Double immunostaining showed that Jagged1-positive cells were myofibroblasts. Notch2 and HES1 were expressed in cholangiocytes of the bile ducts of both rats. Sonic Hedgehog was expressed in the bile ducts of both rats, similarly. Conclusions: A well-balanced and time-sequential expression of the Notch and Hedgehog family in portal tracts might be essential for the normal development of IHBD from E20D until 1W in SD rats, and an imbalanced interaction of these molecules, particularly increased Jagged1 expression in periductal and periportal myofibroblasts and Notch2 expressed in cholangiocytes, may be involved in the formation of bile duct lesions in PCK rats.
\end{abstract}

Key words: intrahepatic bile duct, Notch family, myofibroblasts, ductal plate malformation, Caroli's disease 


\section{Introduction}

Caroli's disease is characterized by the progressive multiple dilatation of intrahepatic bile ducts (IHBD) with congenital hepatic fibrosis (CHF) (1). Most cases of Caroli's disease with CHF show an autosomal recessive inheritance and are associated with autosomal recessive polycystic kidney disease (ARPKD) (2). Polycystic kidney (PCK) rats, an animal model of $\operatorname{ARPKD}(2,3)$, spontaneously develop cystic changes of renal tubules and show a multifocal and irregular dilation of IHBD known as "ductal plate malformation (DPM)" and fibrosis of the portal area $(2,3)$. DPM, an-abnormal biliary and stromal malformation, is characterized by an irregularly shaped and variably dilated duct lumen covered by a low columnar or cuboidal biliary epithelium, bulging or irregular protrusions covered by biliary epithelium into the dilated lumen, and bridge-like structures covered by biliary epithelium in the dilated lumen $(4,5)$, and DPM is characteristically found in patients with Caroli's disease and CHF or in PCK rats (2-5). ARPKD develops as a result of mutations in the causal PKHD1 gene. PCK rats show a deficiency of the pkdh1 protein product, fibrocystin, in the primary cilia, with malformation of primary cilia, which is believed to cause bile duct lesions (6). In addition, the following mechanisms have been also reported to contribute to DPM : disordered cell kinetics of biliary epithelial cells such as the activation of the MEK5-ERK5 cascade $(3,7)$. Basal laminar components and stromal fibrogenesis may also play important roles. Biliary overexpression of plasminogen and tissue-type plasminogen activator leads to the generation of excessive amounts of plasmin, and subsequent plasmindependent lysis of the extracellular matrix molecules may contribute to the formation of DPM $(8,9)$. Several genetic changes involving hepatocyte nuclear factor (HNF)-6, HNF $1 \beta$, and cystin were also reported to be involved in the DPM process (8).

The PCK rat is also characterized by progressive hepatic fibrosis (2). Our previous study revealed two different types of intrahepatic bile ducts: those lined with cuboidal-shaped (C-type) and flat-shaped (F-type) cholangiocytes (11). While the latter was thought to participate in progressive hepatic fibrosis by producing extracellular matrix molecules, the exact mechanisms remain speculative.

Notch signalling is an important pathway in the development of various organs, as well as nerves and blood vessels $(12,13)$. In mammals, Notch1 and Notch 2 transduce signals from their ligands, Jagged1 and Jagged 2, expressed on neighboring cells. This Notch pathway is also important for the normal development 
of intrahepatic bile ducts (IHBD) $(14,15)$, and anomalous biliary development may be directly or indirectly associated with impaired signalling pathways of the Notch family. For example, mutations in Jagged1 cause Alagille syndrome (AGS) characterized by a paucity of bile ducts (16-19). Jagged1 is crucial for the commitment of hepatoblasts to cholangiocytes and also essential for the morphogenesis of IHBD, particularly for the control of the second layer of ductal plates during the remodeling of bile ducts (14). Notch2 signalling pathways also reportedly regulate the differentiation and survival of cholangiocytes and induction of tubulogenesis during the development of IHBD. For example, Tchorz et al reported that expression of the intracellular domain of Notch2 (Notch 2ICD) caused hepatoblasts to differentiate into cholangiocytes, which form additional bile ducts in periportal regions and ectopic ducts in lobular regions, and additional bile ducts in periportal rgions are maintained into adulthood, resulting in an increased number of bile ducts per portal tract (15).

It has been reported that mesenchymal cells in the portal area of mice express Jagged1 and such mesechymal cells are known to express $\alpha$-smooth muscle antigen ( $\alpha$-SMA) (14). In mice whose expression of Jagged1 in portal mesenchymal cells in the portal area has been selectively knocked out, the formation of the tubular structure of the bile ducts is inhibited (17). Taken together, a well-balanced expression and signalling pathway of Jagged1 in periductal mesenchymal cells and Notch2 in cholangiocytes of IHBD might be mandatory for the normal development of bile ducts (14).

So far, there have been many reports on the pathophysiological roles of Notch family signalling pathways in genetic and acquired biliary diseases such as AGS (16-18). For Caroli's disease, however, there have been few studies on the altered signalling pathways of Hedghog (Hh) responsible for DPM, and no studies on Notch family signalling pathways in either the disease itself or its animal model, PCK rats $(20,21)$.

In this study, we examined the roles of Notch and Hedgehog signalling pathways mainly by immunostaining molecules related to these pathways in the normal development of IHBD and also the formation of bile duct lesions in PCK rats. 


\section{Materials and methods}

\section{Animals and collection of liver tissue specimens}

PCK rats were maintained at the Laboratory Animal Institute of Kanazawa University Graduate School of Medicine. Normal (Crl:CD(SD)) rats used as a control were purchased from Charles River Japan (Sagamihara, Japan). Livers were removed from fetal (18 and 20 days of gestation), neonatal, and adult (1day, one-week and 3-week old) rats. After preliminary experiments, more than 5 SD and PCK rats of each age were sacrificed. Liver samples were immersed in a $10 \%$ formalin neutral buffer solution (pH 7.4) and then embedded in paraffin, and also freshly embedded in OTC compound and quickly frozen in liquid nitrogen. More than 20 serial sections, $4 \mu \mathrm{m}$ thick, were cut from the paraffin-embedded and frozen sections. Several of these sections were stained with hematoxylin and eosin (H\&E) and Azan-Mallory, and the remainder were subjected to an immunohistochemical analysis. Parts of the tissue were immediately frozen in liquid nitrogen for use in the reverse transcription-polymerase chain reaction (RT-PCR). In this study, IHBDs and bile ductules were mainly examined. Epithelial cells lining bile ductules and bile ducts are known as cholangiocytes. All animal studies were performed in accordance with the Guidelines for the Care and Use of Laboratory Animals at Takara-machi Campus of Kanazawa University, Kanazawa, Japan.

\section{Immunohistochemistry}

Immunostaining of Jagged 1, Jagged 2, Notch 1, Notch 2, Hes1, $\alpha$-smooth muscle actin ( $\alpha$-SMA), Sonic Hedgehog (Shh), Ki-67 and ssDNA was performed using fresh frozen sections or paraffin-embedded sections of livers from PCK rats and SD rats. Primary antibodies and their sources are shown in Table 1. Frozen sections were fixed with cold acetone followed by blocking of endogenous peroxidase, and incubated with primary antibodies (Jagged 1, Jagged 2, Notch 1, Notch 2, and Hes1) for 1-2h at room temperature. Paraffinembedded sections were deparaffinized and after blocking of endogenous peroxidase, were incubated with individual primary antibodies ( $\alpha$-smooth muscle actin ( $\alpha$-SMA), Sonic Hedgehog (Shh), Ki-67 and ssDNA) for 1-2 hours at room temperature. Then, the sections were incubated with a secondary antibody conjugated to the peroxidase-labeled polymer, Histofine ${ }^{\circledR}$ Simple Stain Rat MAX PO (Nichirei Biosciences Inc., Tokyo, Japan). Color development was performed using 3,3-diaminobenzidine tetrahydrochloride. The sections for

Jagged 1, Jagged 2, Notch 1, Notch 2, $\alpha$-smooth muscle actin ( $\alpha$-SMA), and Sonic Hedgehog (Shh) were 
counter-stained with hematoxylin, lightly. Those for Hes1, Ki-67 and ssDNA were not counterstained. The negative control was evaluated by substitution of the primary antibodies with corresponding non-immunized serum.

\section{Double fluorescecet immunostaining}

Single-color fluorescence immunostaining of Notch 2 , and double-color immunostaining of $\alpha$-SMA and Jagged 1, $\alpha$-SMA and Jagged 2, and $\alpha$-SMA and Notch 1 were performed as follows. Fresh frozen sections were fixed with cold acetone and after the blocking of endogenous peroxidase, incubated overnight at $4^{\circ} \mathrm{C}$ with antibody for Notch 2 or a mixture of antibodies for $\alpha$-SMA and Jagged 1 or $\alpha$-SMA and Jagged 2. The primary antibodies are listed in Table 1. The detection of Notch 2, Jagged 1, and Jagged 2 was performed using a TSA (Tyramide Signal Amplification) ${ }^{\mathrm{TM}}$ kit with Alexa Fluor ${ }^{\circledR} 488$ (Invitrogen, Carlsbad, CA). $\alpha$ SMA was detected with Alexa Fluor ${ }^{\circledR} 594$ secondary antibody (Invitrogen). Nuclei were stained with 4', 6diamidino-2-phenylindole (DAPI).

\section{RT-PCR}

Total RNA was extracted from cryostat sections of the liver using an RNA extraction kit (RNeasy Mini,

Qiagen K. K., Tokyo, Japan) and used to synthesize cDNA with reverse transcriptase (ReverTra Ace, Toyobo Co., Osaka, Japan). Then PCR was performed with KOD DNA polymerase (KOD-Plus or KODPlus-Neo, Toyobo Co.) and primer sets for Notch1, Notch2, Jagged1, Jagged2, HES1, Shh, Gli1, and $\beta$-actin. The sequences of the primers used for PCR are shown in Table 2. The PCR products were subjected to 1\% agarose gel electrophoresis and stained with ethidium bromide.

\section{Image analysis}

ImageJ 1.45s (National Institutes of Health, USA) was used to measure $\alpha$-SMA-positive areas in the liver specimens and for gel densitometry for semi-quantitative RT-PCR.

Labeling index of Ki-67 and ssDNA: Ki-67 expression and ssDNA expression were evaluated in more than 500 cholangiocytes of intrahepatic bile ducts in SD and PCK rats, and the percentage of positive cells was expressed as the Ki-67 LI and ssDNA LI .The former reflects proliferative activities and the latter, apoptosis. The data are shown as the mean of 3 separate measurements, and the error bars show standard deviations. *, $\mathrm{p}<0.05$ in Student's t-test or Welch's t-test. 


\section{Statistics}

The mean and SD was calculated for all parameters. Statistical differences between SD rats and PCK rats were determined using the Student's t-test or Aspin-Welch test, after an analysis of the homogeneity of variance by the F-test. Values of $\mathrm{P}<0.05$ were considered to be statistically significant.

\section{Results}

\section{Pathology of liver and bile ducts in PCK rats}

PCK rats showed abnormal biliary and stromal changes (DPM) characterized by an irregularly shaped and variably dilated lumen covered by low columnar or cuboidal cholangiocytes (1). These DPM patterns were not clearly identifiable at E18D, but became evident at E20D, 1D, 1W and thereafter in PCK rats (Fig.1A,B). Portal tracts were composed of loose connective tissue containing many mesenchymal cells. The loose fibrous stroma was abundant in PCK rats, particularly around the bile ducts and portal veins, in comparison with SD rats. At $3 \mathrm{~W}$, such tissue was significantly less abundant and more hypocellular in both rats. In PCK rats, IHBD was largely classifiable into two types at $3 \mathrm{~W}$ and thereafter: bile ducts lined with cuboidal/columnar cholangiocytes (C-type) and those lined with squamous epithelium similar to mesenchymal cells (F-type) (Fig.2A) (11).

\section{Distribution of a-SMA-positive mesenchymal cells (myofibroblasts) in the portal tracts}

$\alpha$-SMA was constantly expressed in the vascular smooth muscle stages before and after birth in both rats. In SD rats, mesenchymal cells positive for $\alpha$-SMA (myofibroblasts) were also found in the enlarged portal tracts from E20D, 1D and 1W (Fig.1C, Fig.3A). At 3W, there were almost no myofibroblasts in the portal tracts. In PCK rats, myofibroblasts were found in the enlarged portal tracts until $1 \mathrm{~W}$, as seen in SD rats. Myofibroblasts in the portal tracts were more abundant along with the increased amount of loose connective tissue in PCK rats in comparison with SD rats (Fig.1D, Fig.3B). Interestingly, at 3W and later, there were no or few myofibroblasts around C-type bile ducts, similar to SD rats, though myofibroblasts were still present around F-type bile ducts (Fig. 2 B) .

Overall assessment of $\alpha$-SMA-positive areas: Quantitative analysis of $\alpha$-SMA-positive areas in the livers showed that the percentage of $\alpha$-SMA-positive areas increased slightly from E18D to $1 \mathrm{D}$ and decreased 
thereafter in SD rats (Fig.3C). In PCK rats, the percentage of $\alpha$-SMA-positive areas was higher than in SD rats after E20D and peaked $(5.51 \pm 2.41 \%)$ at $1 \mathrm{~W}$, being 2.7 times higher than in SD rats $(2.00 \pm 0.97 \%)$ $(\mathrm{p}<0.05)$. Later, $\alpha$-SMA-positive areas decreased and there was no differences between PCK and SD rats at $3 \mathrm{~W}(1.27 \pm 0.67 \%$ and $0.98 \pm 0.52 \%$, respectively).

\section{Immunohistochemistry: Expression of Notch family-related factors}

Jagged1, Jagged2 and Notch 1: Jagged1 was expressed in the cytoplasm of mesenchymal cells of the portal tracts, particularly around the bile ducts and portal vasculature including portal veins and hepatic arteries at E20D and $1 \mathrm{D}$ and in periportal areas and focally around the portal vasculature at $1 \mathrm{~W}$ in both SD and PCK rats (Fig.4A,B). Double immunostaining showed that a majority of Jagged1-positive cells were also positive for $\alpha$-SMA. Jagged1+ mesenchymal cells in portal tracts of PCK rats outnumbered those of SD rats at E20D, $1 \mathrm{D}$ and $1 \mathrm{~W}$. At $3 \mathrm{~W}$, Jagged 1 was not expressed in mesecnhymal cells in the portal tracts of SD and PCK rats, except for its expression on scattered mesenchymal cells around C-type bile ducts.

Jagged2 and Notch1 were also expressed in the cytoplasm of mesenchymal cells scattered in the portal tracts, particularly around the portal veins at E20D, 1D and 1W in PCK and SD rats (Fig.5A,B). Double immunostaining showed that all Jagged2 and Notch1-positive cells were also positive for $\alpha$-SMA. Jagged2+ and Notch $1+$ mesenchymal cells were not found in the portal tracts after $3 \mathrm{~W}$ in either strain except on a few mesenchymal cells around F-type bile ducts.

Notch2: Notch2 was diffusely expressed in the cytoplasm of hepatocytes and cholangiocytes of both rats, and its expression was rather weak in the hepatocyes (Fig.6A,B). The expression of cytoplasmic Notch2 was similar in cholangiocytes at e20D, 1D, $1 \mathrm{~W}$ and $3 \mathrm{~W}$ in both rats. It was also similarly positive in cholangiocytes of $\mathrm{F}$ and $\mathrm{C}$ type rats. It was focally expressed in mesenchymal cells of the portal tracts.

Hes1: Hes1 was expressed in the nuclei of cholangiocytes of the bile ducts throughout the developmental stages examined, and its expression was slightly stronger in PCK rats in comparison with SD rats. It was expressed similarly in $\mathrm{F}$ type and $\mathrm{C}$ type rats (Table 3).

\section{RT-PCR: mRNA level of Notch family-related factors}

The time-course of mRNA expression of Notch1, Notch2, Jagged1, Jagged2 and Hes1 in liver specimens of both rats is shown in Fig. $7 \boldsymbol{A}$-E. During the fetal period through the age of 3W, the mRNA level of Notch1 in 
PCK rats was similar to that in SD rats. Meanwhile, the level of mRNA of Notch2, Jagged1, and Jagged2 was similar at E18D and E20D in PCK rats and SD rats, but tended to be higher in PCK rats from D1 and/or $1 \mathrm{~W}$.

\section{Expression of Hedgehog family-related factors}

Immunohistochemical expression of Shh: Shh was weakly expressed in the cytoplasm of cholangiocytes in PCK and SD rats (Fig $8 \boldsymbol{A}, \boldsymbol{B})$. It was expressed similarly in cholangiocytes of F type and C type bile ducts of PCK rats.

mRNA level of Shh: RT-PCR showed that the expression of Shh mRNA was very low or undetectable at E18D, E21D, and D1, but increased at 3W in both PCK and SD rats, with no statistically significant difference between the two strains (Fig 8 C $)$.

\section{Expression of Ki67 and ssDNA}

The Ki67 and ssDNA LIs of cholangiocytes of the bile ducts are shown in Fig. 9. While the Ki-67 LI was 6.1 $\pm 7.2 \%, 7.4 \pm 4.4 \%$ and $9.5 \pm 5.7 \%$ in SD rats at $1 \mathrm{D}, 1 \mathrm{~W}$ and $3 \mathrm{~W}$, it was higher significantly in PCK rats $(8.9 \pm 3.9 \%, 15.3 \pm 5.6 \%$ and $17.7 \pm 4.3 \%)$. In contrast, the ssDNA LI was lower, and there was no difference at 1D, $1 \mathrm{~W}$ and $3 \mathrm{~W}$ between PCK and SD rats.

\section{Discussion}

The findings of this study can be summarized as follows: i) $\alpha$-SMA + mesenchymal cells (myofibroblasts) were diffusely distributed in portal tracts of both rat strains while they were abundant in PCK rats. Interestingly, such cells almost disappeared at $3 \mathrm{~W}$ in both strains except around the F-type bile ducts of PCK rats. ii) Jagged1 was expressed in myofibroblasts in portal tracts, particularly around the bile ducts and portal vasculature at E20D and $1 \mathrm{D}$ and in periportal areas at $1 \mathrm{~W}$ in both rats, and Jagged1+ myofibroblasts were rather abundant in PCK rats. Notch2 was expressed in the cholangiocytes in both strains. iii). Shh was similarly expressed in the cholangiocytes of bile ducts in both rats. iv) All the Notch and Hedghog signalling pathway molecules examined were expressed in myofibroblasts and/or cholangiocytes of both rats. v) A well-balanced expression of Notch and Hedgehog-related molecules in portal tracts is important for the normal development of IHBD in SD rats, and an imbalanced expression of Jagged1 in periductal and 
periportal myofibroblasts and interaction with Notch2 on the cholangiocytes may be involved in the formation of bile duct lesions in PCK rats.

Previous studies suggested that cells or factors in the portal areas contributed to the differentiation of hepatoblasts into cholangiocytes and formation of bile ducts (22). IHBD is formed along with the portal veins consisting of endothelial cells and mesenchymal cells, and at the later gestational stage, the interaction of myofibroblasts and cholangiocytes reportedly contributes to the formation of IHBD in mice (14). It was found in this study that the myofibroblasts were distributed in the portal tracts from E20D until 1W in SD rats, suggesting that the interaction of portal myofibroblasts and cholangiocytes at this stage is important in the normal development of IHBD. There are several reports that Jagged1+ mesenchymal cells located in the portal area, particularly in the periportal area where ductal plates and bile ducts are formed and remodeled, were important for the development of IHBD (14). Jagged1+ portal vein mesenchymal cells instruct hepatoblasts expressing Notch2, a receptor of Jagged1, to become cholanigocytes and hence induce the differentiation of hepatoblasts into cholangiocytes $(14,23)$. The signalling pathway of Notch2 in cholangiocytes promotes also fate specification of bile ducts and tubulogenesis in mice (24-26). A hallmark of Notch signalling pathways is the requirement of direct cell-to-cell contact, as both Notch ligands and receptors are integral membrane proteins (27). Thus, IHBD development in the late gestational stage is dependent on Jagged1 and Notch2 expressed in portal tracts (14,16-18).This study also showed that Jagged1 was expressed in periductal and periportal myofibroblasts and Notch2 was expressed in cholangiocytes of SD rats at this stage (E20D - 1W), supporting that a well-balanced and time sequential expression of Jagged1 and Notch2 in the portal tracts and their interaction are essential in the development of IHBD.

Impaired signalling pathways of the Notch family, particularly Notch2 and Jagged1, may be therefore involved in the development of anomalous IHBD (16-18). For example, loss of Jagged1 expression in the periductal mesenchyma due to mutations of Jagged1 leads to AGA with defective bile duct development beyond the initial formation of the ductal plate (20). In contrast, activation of the Notch pathway by forced expression of the Notch intracellular domain in hepatoblasts enhanced cholangiocyte differentiation but suppressed hepatocyte differentiation $(14,23)$. The sustained expression of the intracellular domain of Notch2 (Notch 2ICD) led to additional bile ducts in periportal regions and ectopic ducts in lobular regions, and the 
additional bile ducts in periportal regions were maintained into adulthood, resulting in an increased number of bile ducts per portal tract (15).

PCK rats are known to show DPM (failure of remodeling of bile ducts), and several pathogenetic mechanisms such as altered cell kinetics have been proposed for the DPM $(2,3,11,21,4,21)$. However, there have been no studies on the roles of Notch and Hedghog expression and signalling pathways in cholangiocytes and mesenchymal cells in the livers of PCK rats. It was found in this study that myofibroblasts were abundant in PCK rats in comparison with SD rats at E20D, 1D and 1W, and this abundance was also confirmed by semiquantitative evaluation of $\alpha$-SMA immunostaining. This study showed that the Notch family members examined were expressed in the liver of PCK rats as seen in control rats. However, the number of Jagged1+ myofibroblasts was apparently increased around the bile ducts and portal vasculature and also in the periportal area of PCK rats. Thus, the cholangiocytes of bile ducts of PCK rats at this stage were speculated to be exposed to increased and continuous Jagged1 expression from myofibroblasts. Notch2 expressed in cholangiocytes of bile ducts may interact with Jagged1, suggesting that the myofibroblast-mediated continuous and enhanced activation of Notch 2 - Jagged1 interaction in cholangiocytes might contribute to the bile duct lesions of PCK rats. RT-PCR also showed that the mRNA expression of Notch2 and Jagged1 was up-regulated in PCK rats after birth, supporting the above-mentioned suggestion. The finding that the Ki-67 LI was high in the cholangiocytes of PCK rats, suggested that increased proliferation of cholangiocytes due to the activation of the Notch2/Jagged1 signalling pathway might have contributed to the formation of DPM (2). Taken together, imbalanced interaction of Jagged1 from myofibroblasts with Notch2 in cholangiocytes may be involved in the formation of bile duct lesions in PCK rats. In contrast, Jagged2 was mainly expressed in myofibroblasts around the portal veins, suggesting that Jagged2 was not involved directly in the bile duct lesions of PCK rats at this developmental stage.

Hes1, a downstream target of Notch signalling pathways and regulator of bile duct morphogenesis $(24,25)$, was expressed in the nuclei of cholangiocytes of IHBD at E20D, 1D and 1W, and its expression on cholagiocytes was slightly stronger and its mRNA level was increased in PCK in comparison with SD rats. Interaction of Notch2 in cholangiocytes with Jagged1+ myofibroblasts may activate Hes1 in cholangiocytes, possibly involved in the formation of DPM. Meckel syndrome (MKS) is an another rare autosomal recessive 
disease with DPM and polycystic kidneys (20,21). The gene mutated in type 1 MKS (Mks1) encodes a protein associated with the cilium in vertebrates, and loss of function of mouse Mks1 is an animal model of human MKS with DPM. This bile duct alteration is likely to be the result of the disruption of the Hh signalling pathway. However, the present study showed that Shh was similarly expressed in bile ducts and the mRNA level of Shh was similar in the livers of both rats, suggesting that disruption of the Shh signalling pathway was unlikely involved in the formation of DPM in PCK rats.

Liu et al reported that in the lung and kidney, Notch1 after interaction with its receptor, Jagged1, caused the activation of myofibroblasts (26). Notch1/Jagged1 interaction may also contribute to the enlargement of mesenchymal cells in the portal area during the formation of bile ducts (19). It was found in this study that like Jagged1, Notch1 was expressed in mesenchymal cells in the portal tracts, particularly around the bile ducts and portal veins, in PCK and SD rats and that numbers of Notch1-positive myofibroblasts were increased as seen for Jagged1 in PCK rats in comparison with SD rats, suggesting that mutual interaction of Jagged1 and Notch1 in myofibroblats, might have resulted in an increased number of myofibroblasts themselves around bile ducts.

As for the two different types of bile ducts in PCK rats (11), there were no $\alpha$-SMA-positive myofibroblasts around C-type bile ducts, but F-type bile ducts remained surrounded by myofibroblasts expressing Jagged1 and Notch1. According to our previous study (11), F-type bile ducts showed reduced immunohistochemical expression of the biliary epithelial marker CK 19 and aberrant immunoreactivity for vimentin and fibronectin. In mouse cholangiocytes co-cultured with myofibroblastic hepatic stellate cells, $\mathrm{Hh}$ ligands from these stellate cells promoted EMT of cholagiocytes. The activation of Hedgehog signalling pathways induces immature cholangiocytes to express mesenchymal markers in the bile ducts and also to undergo epithelial mesenchymal transition (EMT), which are likely to contribute to bile duct lesions and fibrosis (27). It was found in this study that Shh was expressed in the cholangiocytes of the F-type and C type bile ducts in PCK rats. Shh expressed in F-type bile ducts may be involved in the EMT of these cholangiocytes, suggesting Hedgehog signalling pathways to be at least partly involved in the EMT of F-type bile ducts of PCK rats. 
In conclusion, the results obtained here suggest that all members of the Notch and Hedgehog families examined were expressed in portal tracts and bile ducts of both PCK and SD rats and that number of Jagged1+ myofibroblasts were increased around the bile ducts of PCK rats. A relatively increased level of Jagged1 expression in myofibroblats along with Notch2 expression in cholangiocytes may be involved in the formation of DPM in PCK rats. More studies are needed to test whether these molecules related to Notch signalling pathways could be a target in the treatment of Caroli's disease. 


\section{References}

1. Caroli J, Couinaud C, Soupault R, Porcher P, Eteve J. A new disease, undoubtedly congenital, of the bile ducts: unilobar cystic dilation of the hepatic ducts. Sem Hop. 1958;34:496-502

2. Sato Y, Ren XS, Nakanuma Y. Caroli's disease: Current knowledge of its biliary pathogenesis obtained from an orthologous rat model. Int J Hepatol 2012;2012:107945.

3. Sanzen T, Harada K, Yasoshima M, Kawamura Y, Ishibashi M, Nakanuma Y. Polycystic kidney rat is a novel animal model of caroli's disease associated with congenital hepatic fibrosis. Am J Pathol 2001;158:1605-12.

4. Desmet VJ. Congenital diseases of intrahepatic bile ducts: variations on the theme "ductal plate malformation". Hepatology. 1992;16:1069-83.

5. Nakanuma Y, Terada T, Ohta G, Kurachi M, Matsubara F. Caroli's disease in congenital hepatic fibrosis and infantile polycystic disease. Liver. 1982;2:346-54.

6. Masyuk TV, Huang BQ, Ward CJ, Masyuk AI, Yuan D, Splinter PL, et al. Defects in cholangiocyte fibrocystin expression and ciliary structure in the PCK rat. Gastroenterology 2003;125:1303-10.

7. Sato Y, Harada K, Kizawa K, Sanzen T, Furubo S, Yasoshima M, Ozaki S, Ishibashi M, Nakanuma Y. Activation of the MEK5/ERK5 cascade is responsible for biliary dysgenesis in a rat model of Caroli's disease. Am J Pathol. 2005;166:49-60.

8. Nakanuma Y, Harada K, Sato Y, Ikeda H. Recent progress in the etiopathogenesis of pediatric biliary disease, particularly Caroli's disease with congenital hepatic fibrosis and biliary atresia. Histol Histopathol. 2010;25:223-35.

9. Yasoshima M, Sato Y, Furubo S, Kizawa K, Sanzen T, Ozaki S, Harada K, Nakanuma Y. Matrix proteins of basement membrane of intrahepatic bile ducts are degraded in congenital hepatic fibrosis and Caroli's disease. J Pathol. 2009;217:442-51.

10. Raynaud P, Tate J, Callens C, Cordi S, Vandersmissen P, Carpentier R, Sempoux C, Devuyst O, Pierreux CE, Courtoy P, Dahan K, Delbecque K, Lepreux S, Pontoglio M, Guay-Woodford LM, Lemaigre FP. A classification of ductal plate malformations based on distinct pathogenic mechanisms of biliary dysmorphogenesis. Hepatology. 2011;53:1959-66. 
11. Sato Y, Harada K, Ozaki S, Furubo S, Kizawa K, Sanzen T, et al. Cholangiocytes with mesenchymal features contribute to progressive hepatic fibrosis of the polycystic kidney rat. Am J Pathol 2007;171:185971.

12. Tien AC, Rajan A, Bellen HJ. A notch updated. J Cell Biol 2009;184:621-9.

13. Bigas A, Robert-Moreno A, Espinosa L. The notch pathway in the developing hematopoietic system. Int J Dev Biol 2010;54:1175-88.

14. Suzuki K, Tanaka M, Watanabe N, Saito S, Nonaka H, Miyajima A. P75 neurotrophin receptor is a marker for precursors of stellate cells and portal fibroblasts in mouse fetal liver. Gastroenterology 2008;135:270-281.e3.

15. Tchorz JS, Kinter J, Müller M, Tornillo L, Heim MH, Bettler B. Notch2 signaling promotes biliary epithelial cell fate specification and tubulogenesis during bile duct development in mice. Hepatology. 2009;50:871-9.

16. Antoniou A, Raynaud P, Cordi S, Zong Y, Tronche F, Stanger BZ, et al. Intrahepatic bile ducts develop according to a new mode of tubulogenesis regulated by the transcription factor SOX9. Gastroenterology 2009;136:2325-33.

17. Hofmann JJ, Zovein AC, Koh H, Radtke F, Weinmaster G, Iruela-Arispe ML. Jagged1 in the portal vein mesenchyme regulates intrahepatic bile duct development: Insights into alagille syndrome. Development 2010;137:4061-72.

18. Crosnier C, Attié-Bitach T, Encha-Razavi F, Audollent S, Soudy F, Hadchouel M, et al. JAGGED1 gene expression during human embryogenesis elucidates the wide phenotypic spectrum of alagille syndrome. Hepatology 2000;32:574-81.

19. Hofmann JJ, Zovein AC, Koh H, Radtke F, Weinmaster G, Iruela-Arispe ML. Jagged1 in the portal vein mesenchyme regulates intrahepatic bile duct development: Insights into alagille syndrome. Development 2010;137:4061-72.

20. Dowdle WE, Robinson JF, Kneist A, Sirerol-Piquer MS, Frints SG, Corbit KC, Zaghloul NA, van Lijnschoten G, Mulders L, Verver DE, Zerres K, Reed RR, Attié-Bitach T, Johnson CA, García-Verdugo JM, Katsanis N, Bergmann C, Reiter JF. Disruption of a ciliary B9 protein complex causes Meckel syndrome. 
Am J Hum Genet. 2011;89:94-110.

21. Weatherbee SD, Niswander LA, Anderson KV. A mouse model for Meckel syndrome reveals Mks1 is required for ciliogenesis and Hedgehog signaling. Hum Mol Genet. 2009;18:4565-75.

22. Shiojiri N. Development and differentiation of bile ducts in the mammalian liver. Microsc Res Tech. 1997;39:328-35.

23. Tanimizu N, Miyajima A. Notch signaling controls hepatoblast differentiation by altering the expression of liver-enriched transcription factors. J Cell Sci. 2004;117:3165-74.

24. Kodama Y, Hijikata M, Kageyama R, Shimotohno K, Chiba T. The role of notch signaling in the development of intrahepatic bile ducts. Gastroenterology. 2004;127:1775-86.

25. Liu X, Li J, Xiong J, Li M, Zhang Y, Zheng Q. Notch-dependent expression of epithelial-mesenchymal transition markers in cholangiocytes after liver transplantation. Hepatol Res. 2012 (in press)

26. Liu T, Hu B, Choi YY, Chung M, Ullenbruch M, Yu H, et al. Notch1 signaling in FIZZ1 induction of myofibroblast differentiation. Am J Pathol 2009;174:1745-55.

27. Omenetti A, Choi S, Michelotti G, Diehl AM. Hedgehog signaling in the liver. J Hepatol 2011;54:366-73. 


\section{Figure legends}

Fig.1. Bile duct lesions of PCK and SD rats (1 day-old). A: There was connective tissue where bile ducts were located. Portal area of SD rats. B: Dilatation of the ductal plate was evident and there was loose and cellular connective tissue in the portal tract, including around the bile ducts, in the PCK liver. BD, bile duct; $\mathrm{PV}$, portal vein. $\mathrm{Bar}=100 \mu \mathrm{m} . \mathrm{H} \& \mathrm{E}$.

Fig. 2. Phenotypes of intrahepatic bile ducts in PCK rats (3 weeks old) and d istribution of myofibroblasts. A: Two types of intrahepatic bile duct, those lined by flat-shaped (F-type) cholangiocytes and by cuboidalshaped (C-type) cholangiocytes, were found in 3-week-old PCK rats. Bar $=100 \mu \mathrm{m}$. HE. B: $\alpha$-smooth muscle actin ( $\alpha$-SMA)-positive myofibroblasts were distributed around the F-type bile ducts but not around C-type bile ducts and in other parts of portal tracts. Bar $=100 \mu \mathrm{m}$. Immunohistochemical staining for $\alpha$-SMA counterstained by hematoxylin.

Fig 3. Immunohistochemical staining for $\alpha$-smooth muscle actin ( $\alpha$-SMA) (red) in the liver of PCK rats and SD rats. A,B: Expression of $\alpha$-SMA in portal areas through the development of bile ducts in SD rats (A) and PCK rats (B). In SD rats (A), $\alpha$-SMA-positive mesenchymal cells (myofibroblasts) were found in the portal tracts, particularly around the bile ducts and portal veins, from embryonic day 20 (E20D) until postnatal week $1(1 \mathrm{~W})$. Later, at 3 weeks after birth $(3 \mathrm{~W})$, vascular smooth muscles remained present, though the expression of $\alpha$-SMA in mesenchymal cells in portal tracts including periductal areas disappeared. In PCK rats (B), until 1 week after birth, the distribution of $\alpha$-SMA expression was similar to that in SD rats. However, $\alpha$-SMA-positive regions were abundant in comparison with those in SD rats. In addition, in PCK rats, F-type bile ducts (arrow) at 3 weeks after birth were still surrounded by $\alpha$-SMA-positive cells. Bar $=100$

$\mu \mathrm{m} . \mathbf{C}$ : Comparison of $\alpha$-SMA-positive areas in the liver specimens between SD and PCK rats (percentage of positive areas) according to age showed that $\alpha$-SMA-positive areas of PCK rats $(\bullet)$ were abundant in comparison with SD rats (०); PCK rats showed a peak at $1 \mathrm{w}$ after birth. However, there was a significant difference at $1 \mathrm{~W}(\mathrm{p}<0.05)$. The data are shown as the mean of 5 separate measurements, and the error bars show standard deviations. 
Fig 4. Double immunofluorescence staining for Jagged1 (green) and $\alpha$-smooth muscle actin ( $\alpha$-SMA) (red) in SD rats (A) and PCK rats (B) at 1day and 1week of age.

A: Expression of Jagged1 was found in mesenchymal cells of the portal tracts, particularly around portal veins and also bile ducts at $1 \mathrm{D}$ and in periportal areas and focally around bile ducts and blood vessels at $1 \mathrm{~W}$ in SD rats. A majority of Jagged1-positive cells were also positive for $\alpha$-SMA. B: Jagged1 was expressed in mesenchymal cells of the portal tracts, particularly around blood vessels and also bile ducts at 1D and in periportal areas and focally around blood vessels at $1 \mathrm{~W}$ in PCK rats. A majority of Jagged1-positive cells were also positive for $\alpha$-SMA. The number of Jagged $1+$ cells and $\alpha$-SMA+ cells of PCK rats outnumbered that of SD rats. BD, bile duct; PV, portal vein. Nuclear staining was performed with DAPI (in blue).

Fig. 5. Double immunofluorescence staining for Jagged 2 (green) and $\alpha$-smooth muscle actin ( $\alpha$-SMA) (red) in SD rats (A) and PCK rats (B) at 1day and 1week of age. A: Expression of Jagged2 was mainly found in mesenchymal cells of the portal tracts, particularly around portal veins at 1D and 1W. B: Jagged1-positive cells were also distributed in the portal tracts, particularly around the portal vein as seen in SD rats. BD, bile duct; PV, portal vein. Nuclear staining was performed with DAPI (in blue).

Fig. 6. Immunohistochemical staining for Notch2 in the liver of SD rats (A) and PCK rats (B) at 1week of age. Notch2 was weakly expressed in the cytoplasm of hepatocytes and evidently expressed in that of cholangiocytes of both rats. $\mathrm{BD}$, bile duct; $\mathrm{PV}$, portal vein. $\mathrm{Bar}=100 \mu \mathrm{m}$.

Fig. 7. Time-course of mRNA expression of Notch1 (A), Notch2 (B), Jagged1 (C), Jagged2 (D) and Hes1 (E) measured by RT-PCR in liver specimens of SD (०) and PCK $(\bullet)$ rats. During the fetal period through to the age of $3 \mathrm{~W}$, there was no difference in the mRNA level of Notchl between PCK rats and SD rats. Meanwhile, while the levels of mRNA for Notch2, Jagged1, and Jagged2 were similar at E18D and E20D in PCK and SD rats, they tended to be slightly higher in the PCK rats from D1 and/or 1W. *, $<$ P0.05 
Fig. 8. Expression of Sonic Hedgehog in the livers of PCK and SD rats.

A, B: Immunohistochemical staining showed that Shh was expressed similarly in the cytoplasm in bile ducts of SD rats (A) and PCK rats (B) at $1 \mathrm{~W}$ (bile ducts: arrows).

C: RT-PCR analysis of the mRNA expression of Shh in the livers of PCK rats $(\bullet)$ and SD rats (०) from embryonic day 18 until postnatal week 10 . The values are shown as ratios with respect to $\beta$-actin expression. The mRNA level of Shh was similar in SD rats and PCK rats. The data are shown as the mean of 5 separate measurements, and the error bars show standard deviations.

Fig 9. Immunohistochemical study of ki-67 and ssDNA in the bile duct epithelium of PCK rats and SD rats.

A,B: Labeling indexes (LIs) of ki-67 and ssDNA in cholangiocytes of the bile ducts of PCK rats (O) and SD rats $(\bullet)$ are shown. The ki-67 LI was significantly higher in PCK rats (०) than in SD rats (•) (A). Meanwhile, there was no difference in the ssDNA LI between PCK rats and healthy rats. The data are shown as the mean of 5 separate measurements, and the error bars show standard deviations. *, $\mathrm{p}<0.05$ with Student's t-test or Welch's t-test. 
Table 1. Primary Antibodies Used for the Immunohistochemical Analysis

\begin{tabular}{lllll}
\hline Antibody & Species & Supplier & Dilution & Specimen \\
\hline Jagged1 (H-114) & Rabbit & SCB & $1 / 50$ & Frozen \\
Jagged2 (H-143) & Rabbit & SCB & $1 / 50$ & Frozen \\
Notch1 (C-20) & Rabbit & SCB & $1 / 50$ & Frozen \\
Notch2 (LS-B719) & Rabbit & Lifespan & $1 / 50$ & Frozen \\
HES1 (H-140) & Rabbit & SCB & $1 / 50$ & Frozen \\
Alpha-Smooth muscle actin & Mouse & Dako & $1 / 100$ & Paraffin (MW) \\
Sonic Hedgehog (GT15032) & Goat & Neuromics & $1 / 50$ & Paraffin (MW) \\
Ki-67 & Mouse & Dako & $1 / 100$ & Paraffin (MW) \\
ssDNA & Rabbit & Dako & $1 / 100$ & Paraffin (MW) \\
\hline
\end{tabular}

SCB, Santa Cruz Biotechnology, Inc. (Santa Cruz, CA); Lifespan, LifeSpan BioSciences, Inc. (Seattle, WA); DAKO, DakoCytomation (Glostrup, Denmark); Neuromics, Neuromics (Edina, MN); MW, Antigen retrieval was performed by microwaving in 10 $\mathrm{mmol} / \mathrm{L}$ citrate buffer $\mathrm{pH}$ 6.0. 
Table 2. Sequences of the Primers Used

\begin{tabular}{lll}
\hline Gene & Sequences & Product size \\
\hline Beta-actin & 5'-ACCTTCAACACCCCAGCCATGTACG-3' & 698 \\
& 5'-CTGATCCACATCTGCTGGAAGGTGG-3' & \\
\hline Jagged1 & 5'-CCAGAATGGCAACAAAACCT-3' & \multirow{2}{*}{459} \\
& 5'-CAGCCTGGAGAACACTCACA-3' & \\
\hline Jagged2 & 5'-AACGACTTCTTCGGCCACTA-3' & \multirow{2}{*}{465} \\
& 5'-AAAGCCAGACAGCACTTCGT-3' & \\
\hline Notch1 & 5'-GTTTGTGCAAGGATGGTGTG-3' & \multirow{2}{*}{482} \\
& 5'-GCACTCGTTGACCTCAGACA-3' & \\
\hline Notch2 & 5'-GAGTGTCTGAAGGGCTACGC-3' & \\
& 5'-TGCAGATGCAGGTGTAGGAG-3' & \multirow{2}{*}{430} \\
\hline HES1 & 5'-GCTGGAGAAGGCAGACATTC-3' & \\
\hline Sonic Hedgehog & 5'-CTGGCCAGATGTTTTCTGGT-3' & \multirow{2}{*}{117} \\
\hline
\end{tabular}


Table 3 Summary of Immunohistochemical expression of Notch1/2 and Jagged1/2 in the liver of the PCK rat

\begin{tabular}{|c|c|c|c|c|}
\hline $\begin{array}{l}\text { Cell } \\
\text { components }\end{array}$ & Notch1 & Notch2 & Jagged 1 & Jagged2 \\
\hline Cholangiocyte & - & ++ & - & - \\
\hline Hepatocyte & - & + & - & - \\
\hline Myofibroblast & ++ & + & ++ & ++ \\
\hline
\end{tabular}

-, no remarkable; +, slightly expressed; ++, markedly expressed. 
Fig.1

A

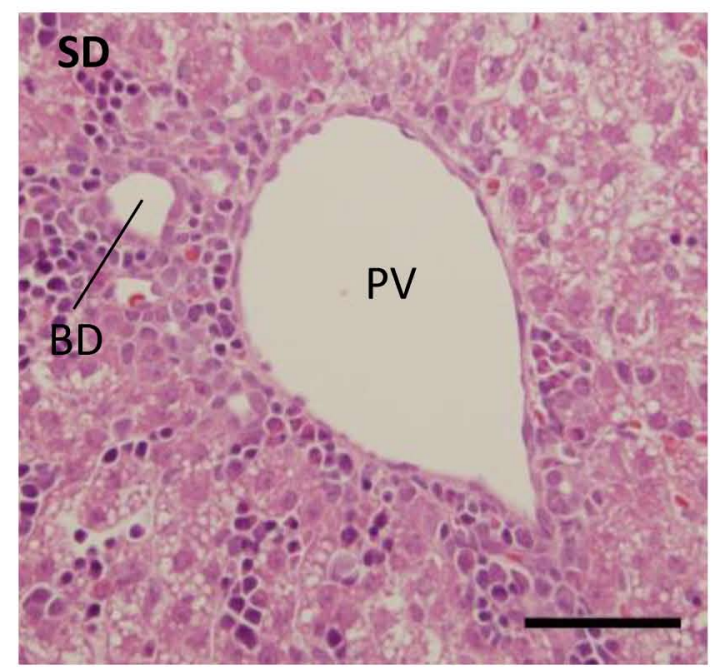

C

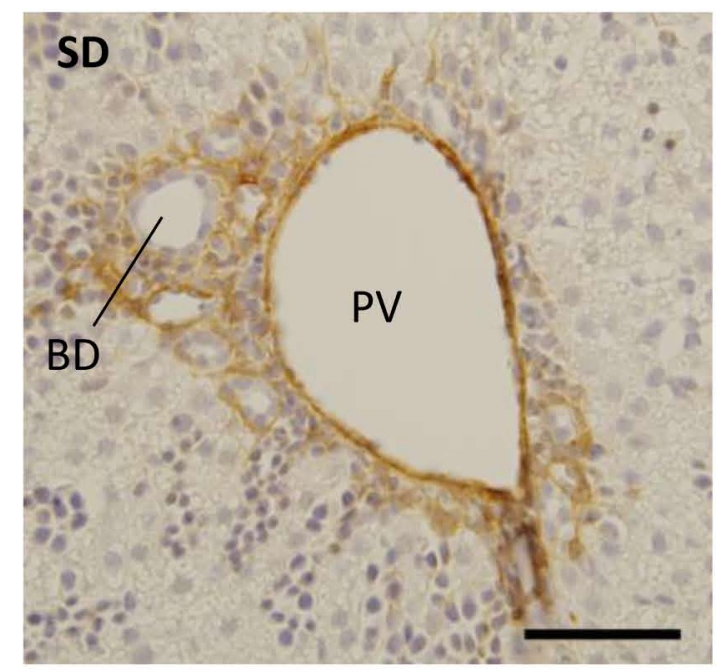

B

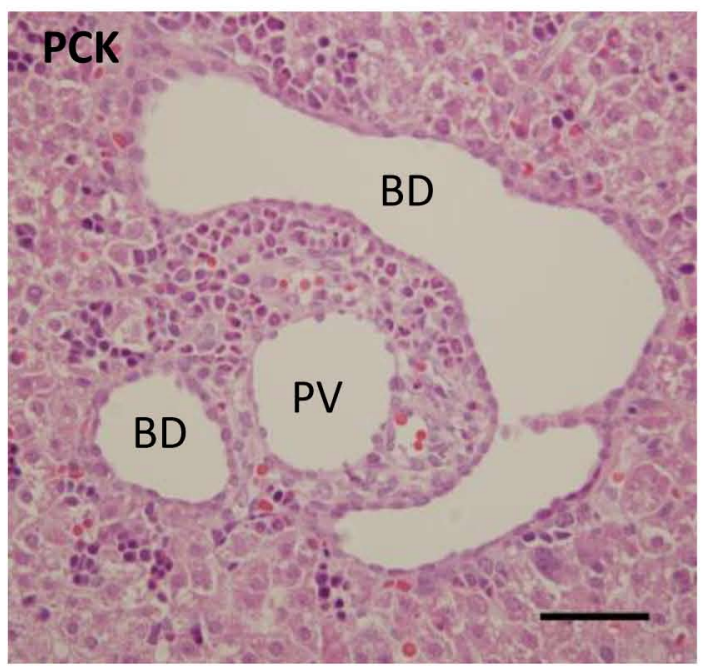

D

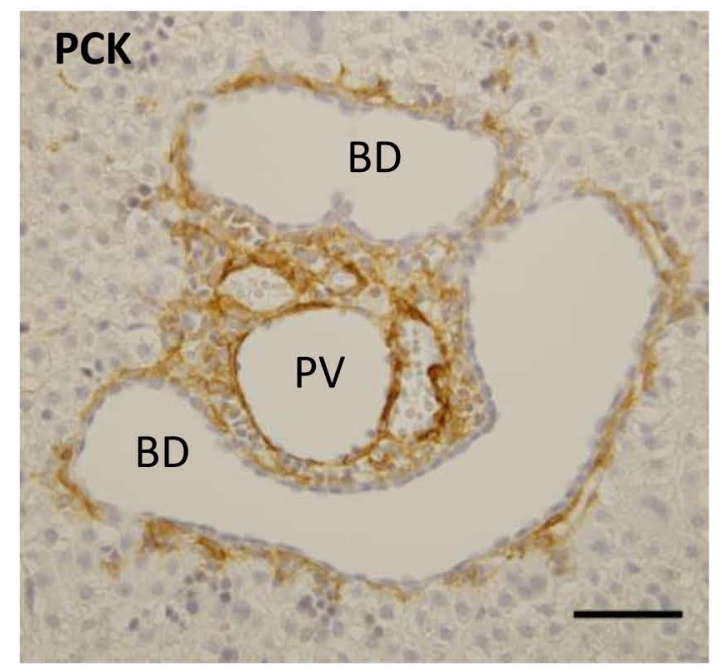


Fig.2

A

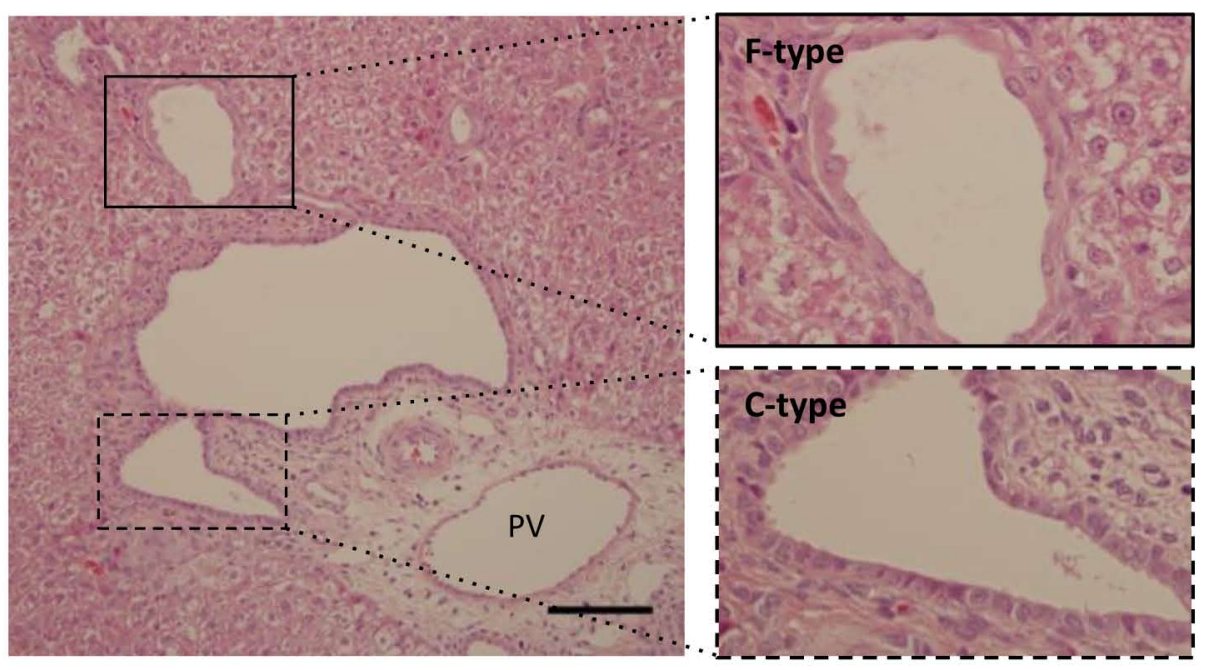

B

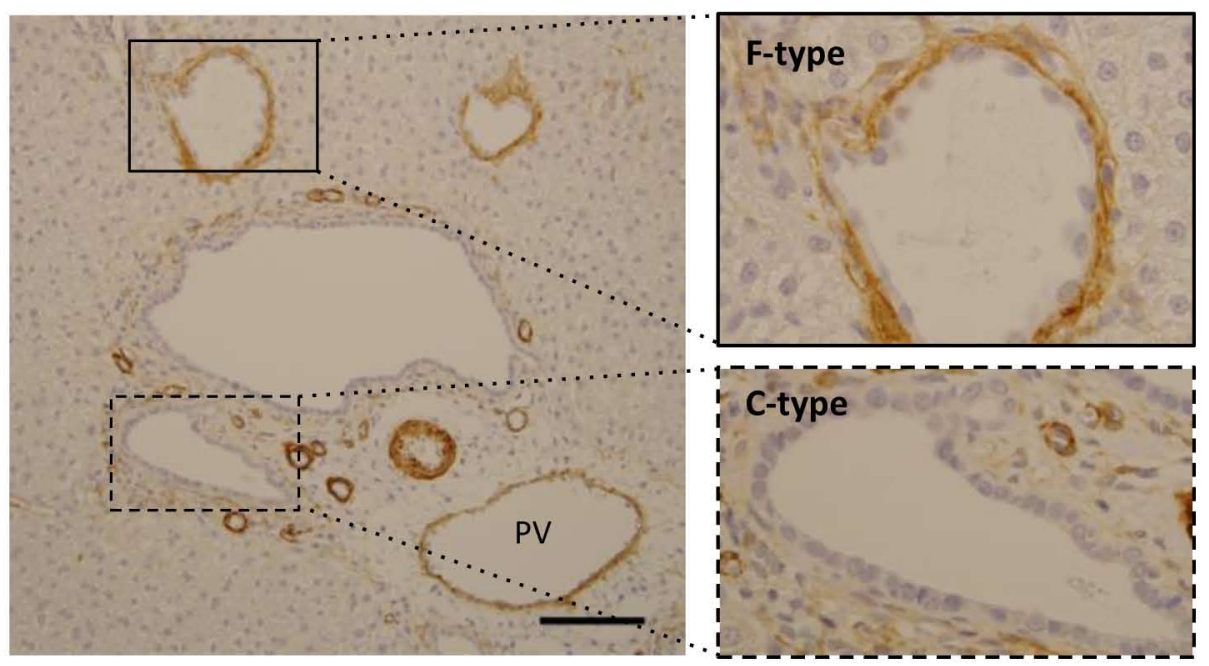


Fig.3

A
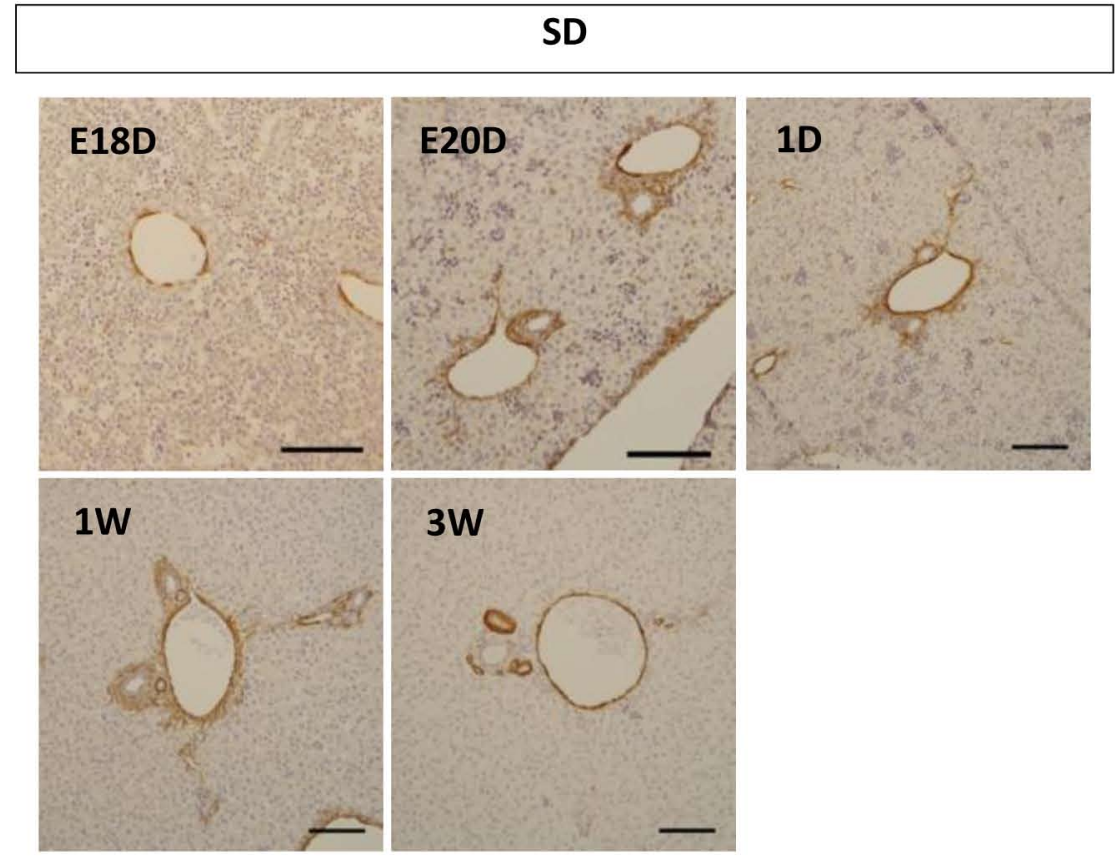

$3 w$

B
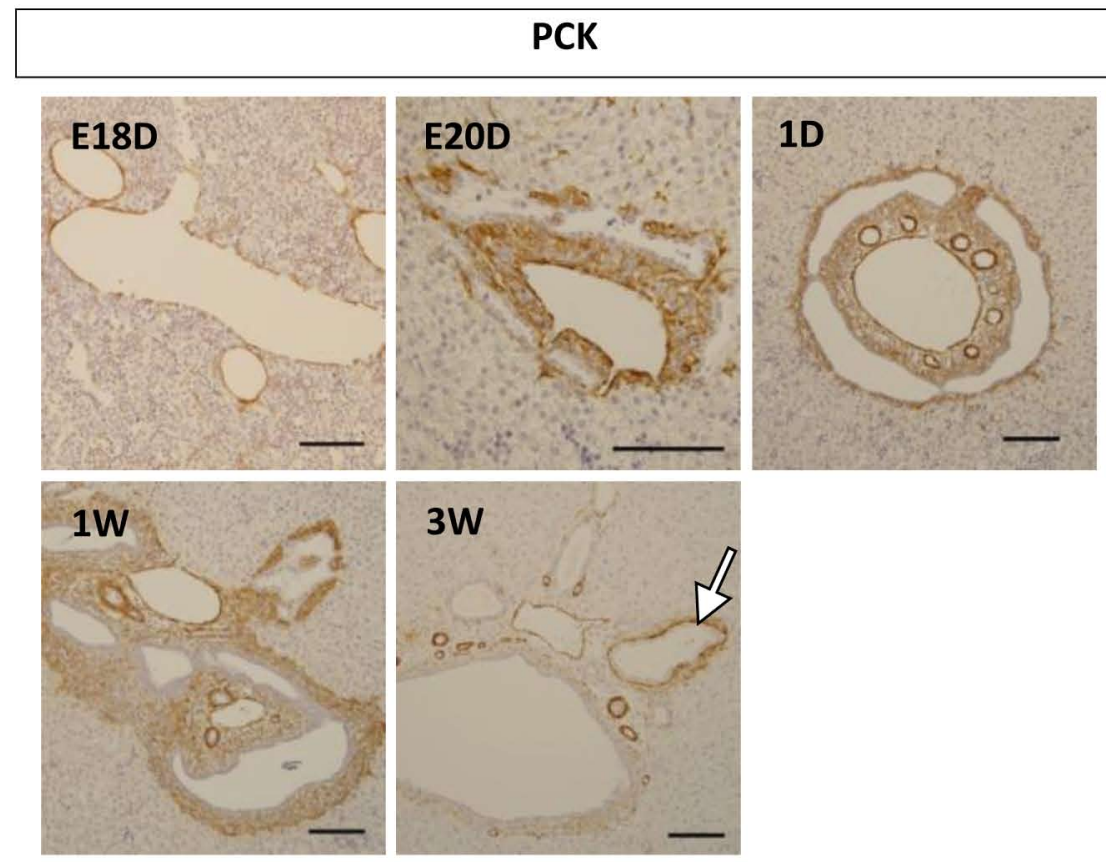

C

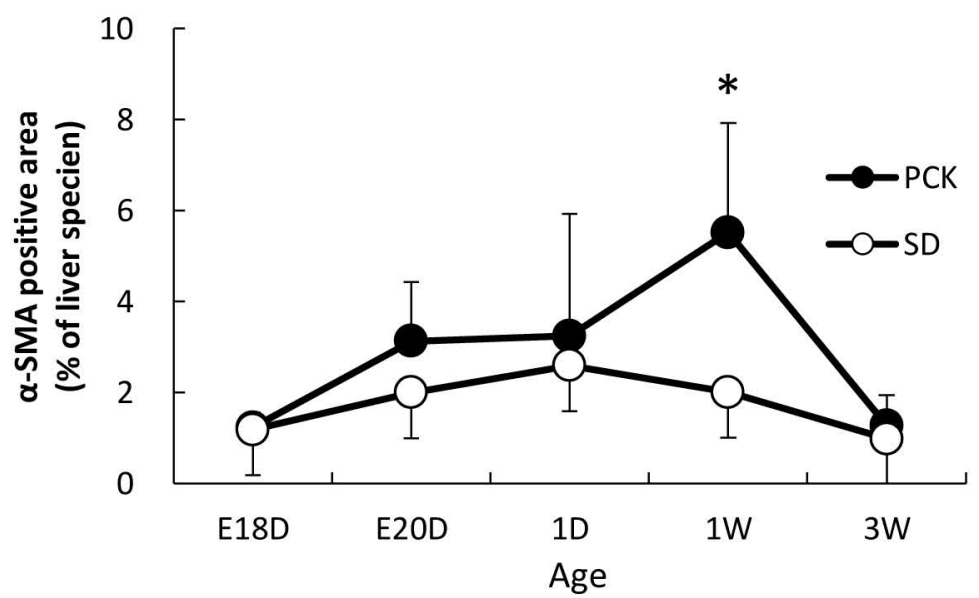


Fig.4
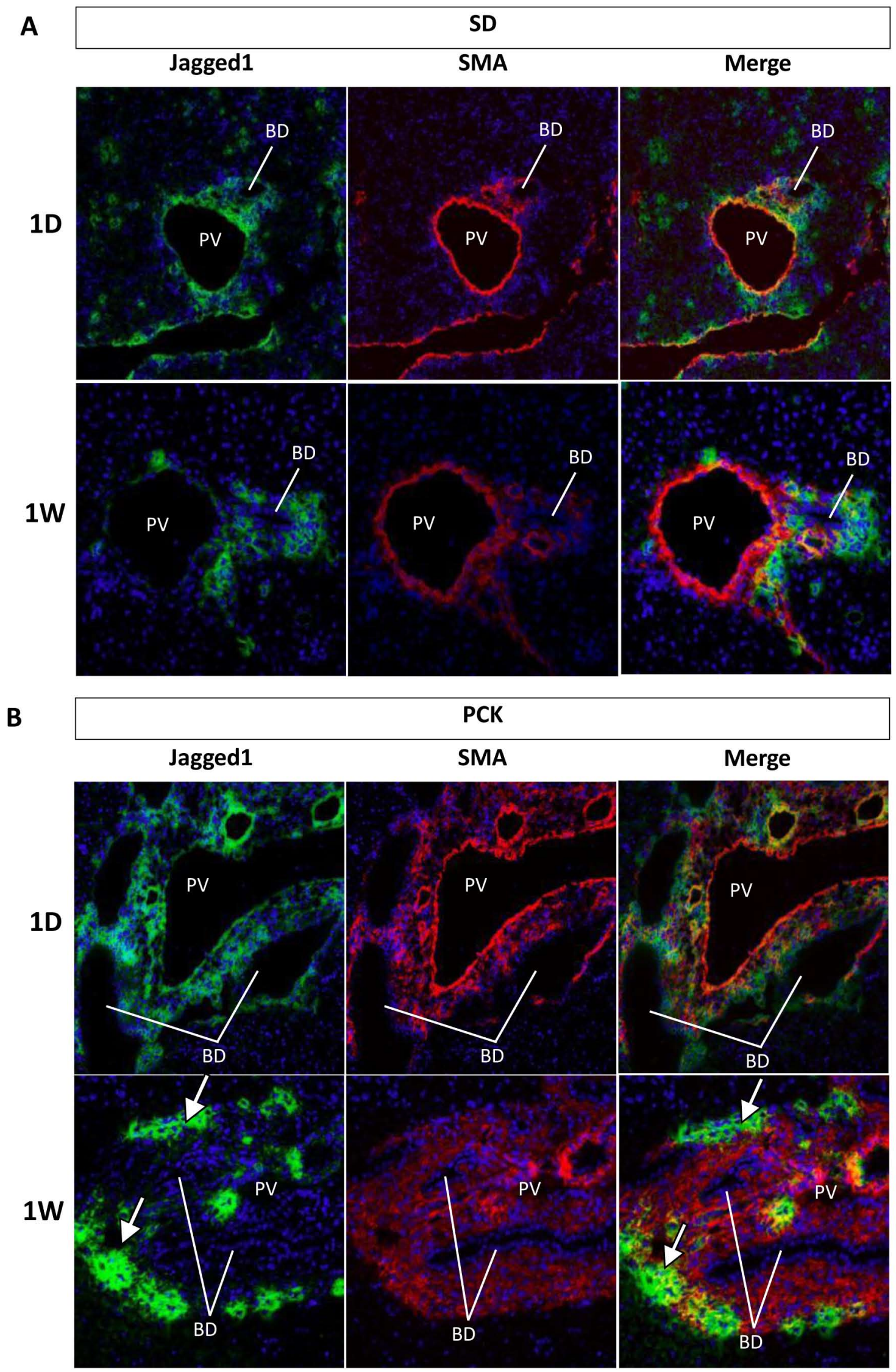
Fig.5
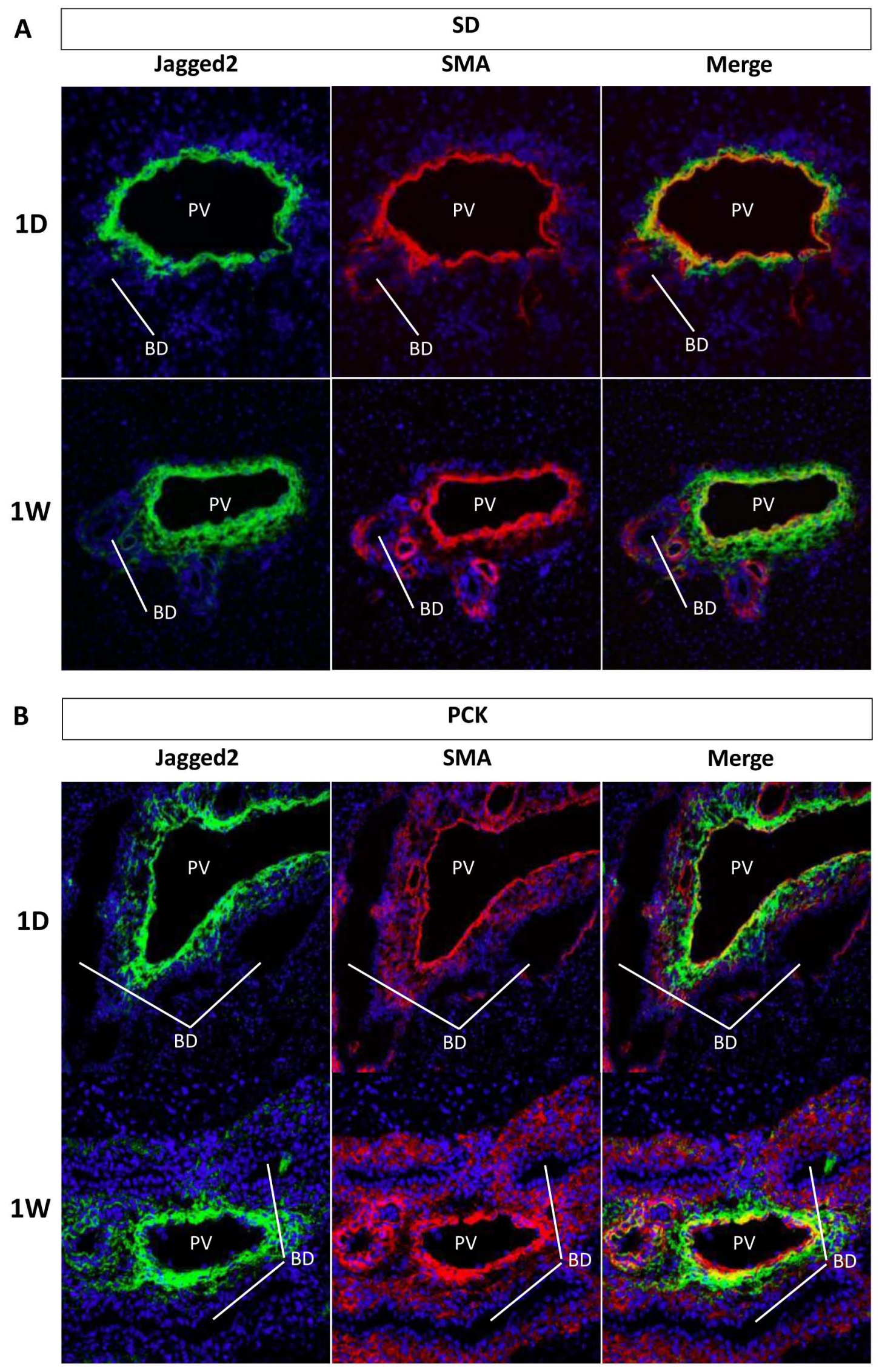
Fig.6

A

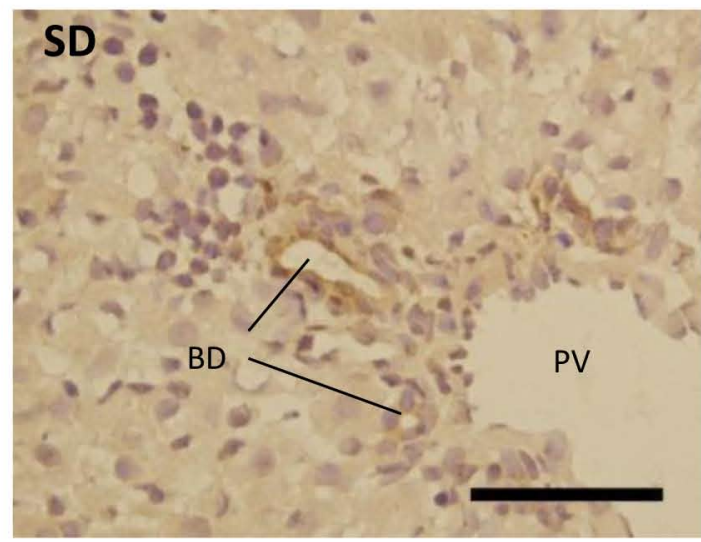

B

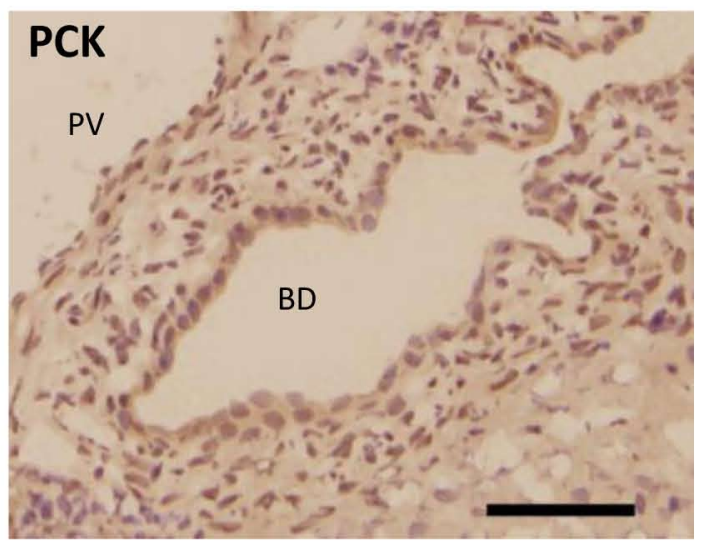


Fig. 7

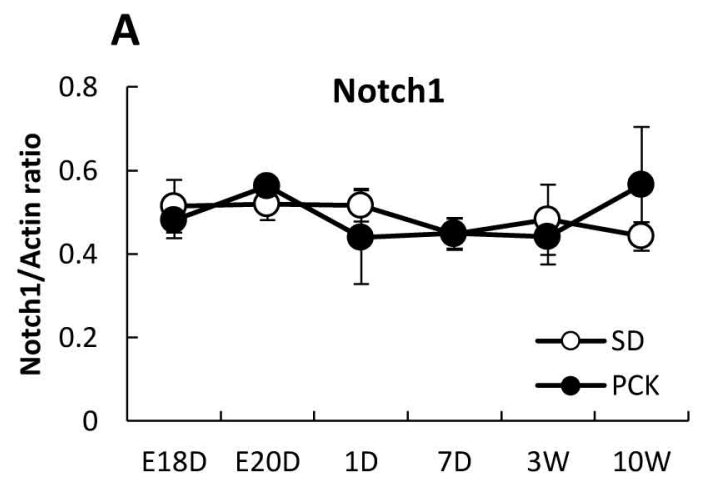

\section{C}

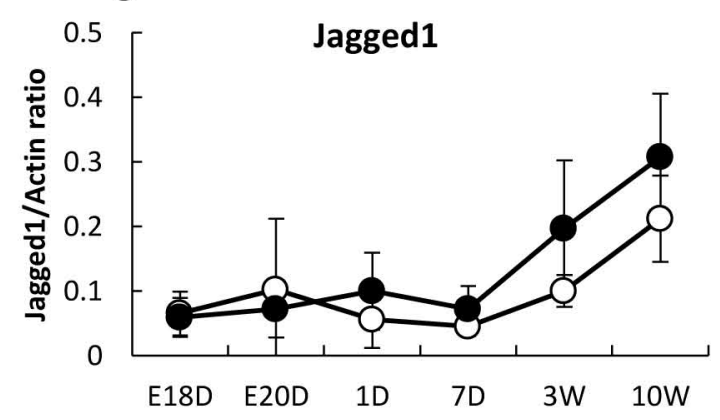

\section{E}

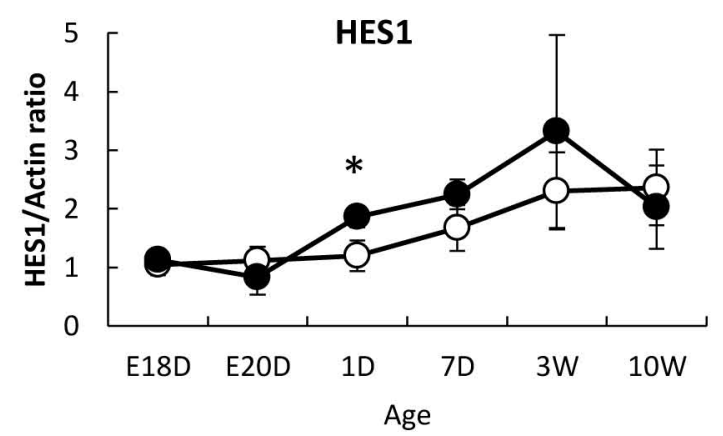

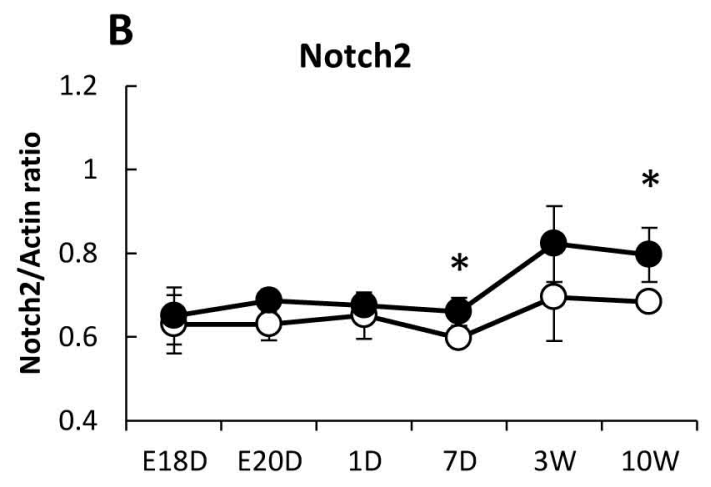

D

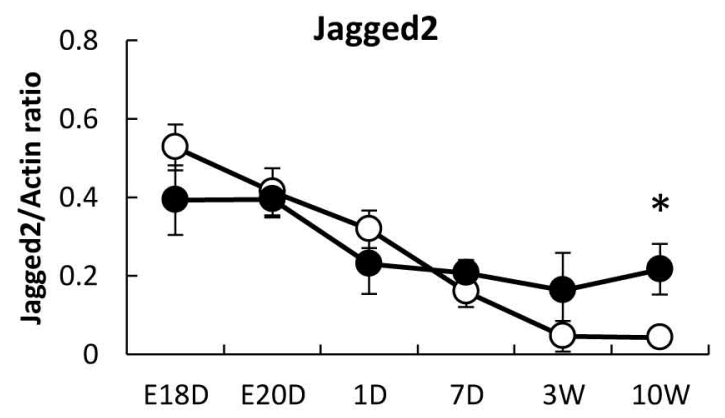


Fig.8

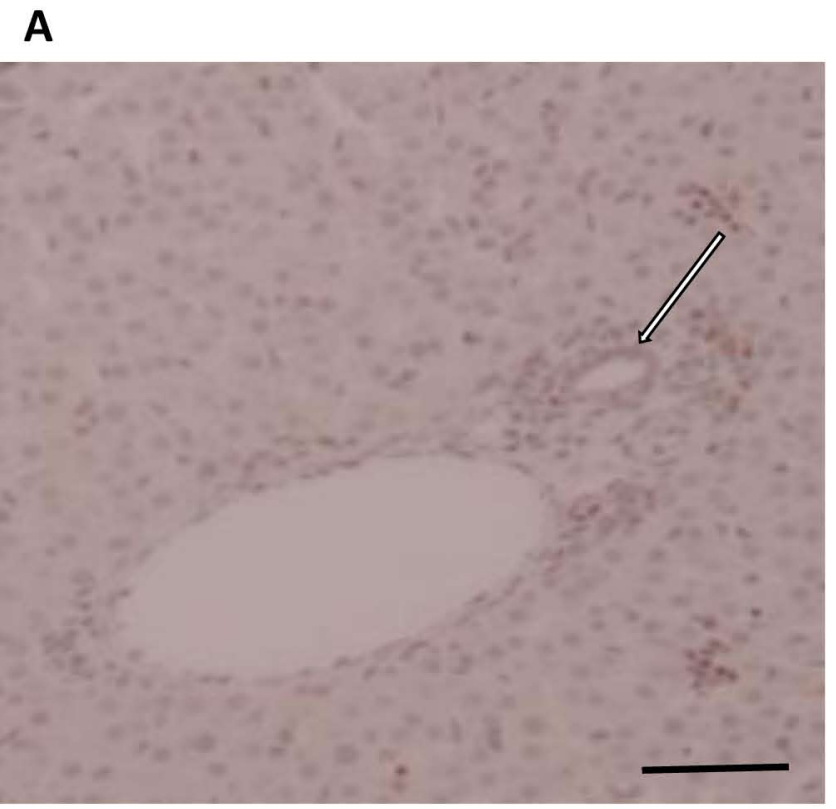

B

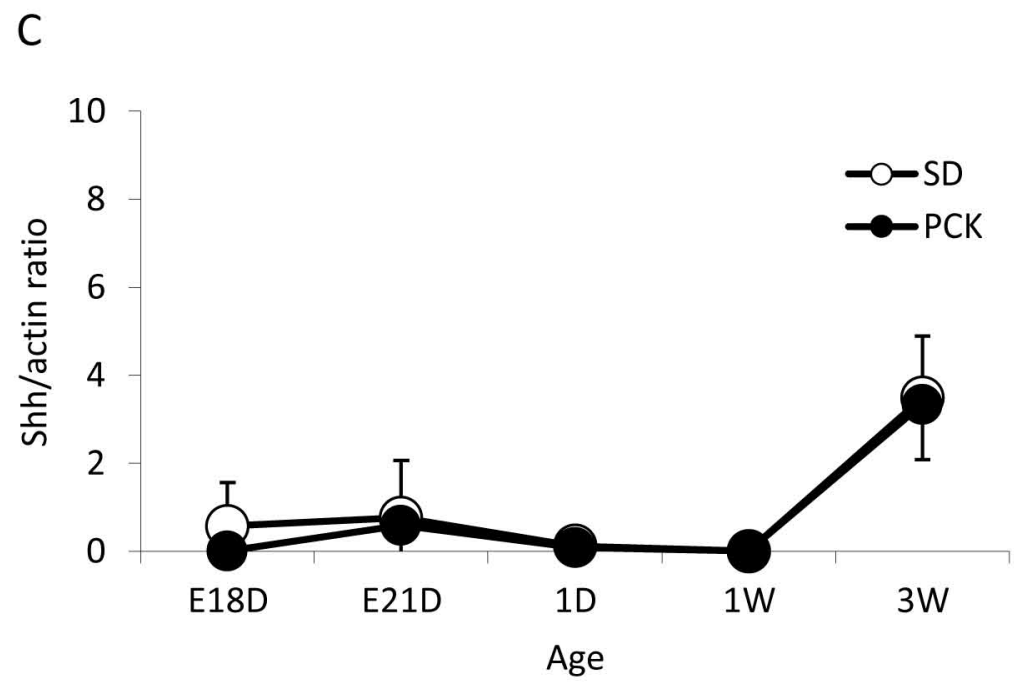


Fig.9

A

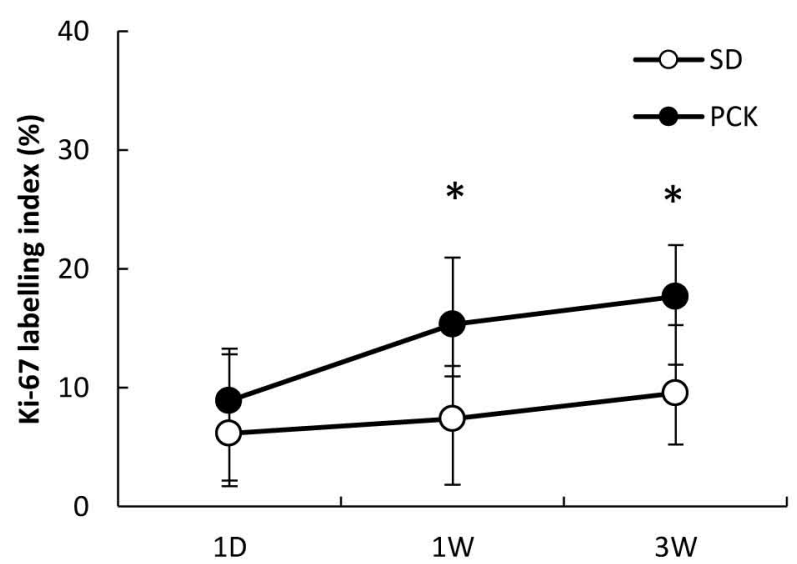

B

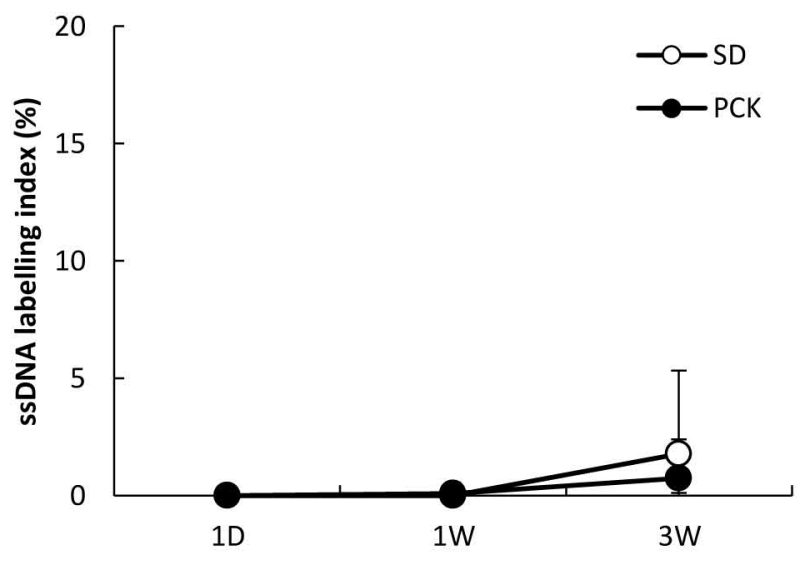

